\title{
New "Graphiton" Model: a Computational Discrete Space, Self-Encoded as a Trivalent Graph
}

\author{
Raymond Aschheim \\ Polytopics Research Institute \\ Issy-les-Moulineaux 92130, France \\ Tel: 33-685-662-215Ｅ-mail: raymond@aschheim.com \\ Smain Femmam (Corresponding author) \\ University of Haute Alsace UHA \\ Mulhouse 68093, France \\ $\&$ \\ Polytechnic Engineering School EPF Labs \\ Sceaux 92330, France \\ Tel: 33-643-668-441Ｅ-mail: femmam@ieee.org \\ M. Faouzi Zerarka \\ Polytechnic Engineering School EPF Labs \\ Sceaux 92330, France \\ E-mail: faouzi.zerarka@gmail.com
}

Received: September 15, 2011

Accepted: December 5, 2011

Published: January 1, 2012

doi:10.5539/cis.v5n1p2

URL: http://dx.doi.org/10.5539/cis.v5n1p2

\begin{abstract}
The new graphiton models described here are trivalent graphs which encode topologically binary information. They permit defining intrinsic discrete spaces which constitute supernode crystals. Besides encoding its own metric, the model supports disturbances due to fault tolerance through the redundancy of information in the paths of connection between supernodes. Coming from theoretical physics, they may find applications in network management and artificial intelligence. For the first time, an information system structure, rich enough to model the universe itself, but relying ultimately on set theory, traverses set theory, topology, information theory, graph theory, geometry, algebra, theoretical physics and even computer and network science, in a logical straightforward and elegant way.
\end{abstract}

Keywords: Network coding and theory, Complexity theory, Quaternion, Lattices

\section{Introduction}

We present here a new type of model particularly suited to solving physical problems in non-Euclidean spaces. These spaces may mix the concepts of proximity (such as geographic information systems), topological notions (such as social networks), information concepts, and algebraic concepts (such as the invariance of certain local symmetries).

Our model was developed to provide a framework for digital physics (Aschheim, 2008). It is a conceptual breakthrough in the definition of a space based on set theory (Aschheim, 2009a). We can compute gravitational field (Aschheim, 2010) from its metrics. Here we specify its formalism in information theory, opening up applications to networks.

This model is a complex system on several levels, where the fundamental level is that of a trivalent graph.

The second level is the encoding of information in the topological graph structure. 
The third is the encoding of local symmetries and algebraic properties.

The fourth is the emergence of a metric, a dimensionality and a curvature of the assembly of subgraphs, as supernodes in a network (Femmam, 2012) of crystalline type.

It is possible to work at every level of this model by maintaining a good coherence. We optimize at a fundamental level the simplicity of the structure, while allowing the emergence of great complexity in the modeled system.

This family of models is designed to provide a framework for a new theory of everything, unifying in theoretical physics, the phenomena of quantum physics and our knowledge of the standard model with gravity and the conception of a space according to general relativity. And we are dealing with discrete model instead of continuous one. There is still debate whether the nature of the universe is discrete or continuous. Some even speculate that the reality is the alliance of two (Chamseddine \& Connes, 2010). "We examine the hypothesis that space-time is a product of a continuous four-dimensional manifold times a finite space."

Others postulate a discrete system (and possibly finite) is sufficient for the emergence of an apparent continuity, which is an approximation of the discrete. The main result of the present work is the demonstration that a phenomenon hitherto treated as continuous course, emerges naturally in our discrete model: gravity. As it emerges from a simple graph, we call it "graphitation". A similar word game has already been used for an approach to quantum physics in a graph, called "Quantum Graphity" (Markopoulou \& Smolin, 2004; Konopka, Markopoulou \& Severini, 2008). But the approaches are different; physicists have built several discrete models, graphs or networks, to implement continuous physical equations, by keeping the current models in discreet format. These approaches are top-down, as they depart from known results, to find a consistent underlying structure.

Our approach is bottom up, starting with a simple model ultimately (in fact the level of set theory, the foundation of mathematics), and constructing according to a principle of economy, said "Okham's razor principle" (de Ockam, a) (Rursus absque necessitate et utilitate est pluralitas fugienda) (de Ockam, b) a layered model to the more complex reality.

\section{Graphitation, Bottom-Up Approach}

In our bottom-up approach, we start with a trivalent undirected graph.

\subsection{Construction}

\subsubsection{Definition}

A graphiton model is a trivalent graph checking at least two properties: a quasi-invariance on a large scale (a large number of nodes in the graph, evenly spaced, are topologically equivalent if one neglects a small number of disturbances), a characterization of local geometry at small-scale by the presence of triangular loops encoding bit 1.

Graphiton, a basic element of a graphiton model, is simply a node with three ends, each connected to another graphiton.

So, while any totally trivalent graph is a blend of graphitons, for it to be a model, it must satisfy the properties of global quasi-invariance and local geometric encoding.

A graphiton model can be interpreted in different scales. We will distinguish three scales.

Level 0 is the fundamental level, in which the model is a simple undirected trivalent graph, where any node or connection doesn't carry additional information.

Level 1 is obtained from level 0 by adding a binary property in each node; each node carries a bit, taking the value 0 or value 1 .

The equivalence between Level 1, informed graph, and level 0 , is ensured by the transformation T2 (see Figure 3) by Alexander (Alexander \& Briggs, 1926), which replaces each node with a value of 1 at level 1 as a triangle consisting of three nodes at 0 , and does not change the nodes on the value 0 to level 1 .

If the graph is information homogeneous, id-est. contains as much bit 0 as bit 1 at level 1 , while its equivalent in level 0 will double its number of nodes (and therefore connections).

Unless explicitly stated otherwise, we always work on information homogeneous graphs. 
Our models have a natural encoding and regular, homogeneous information, which can be seen as physically optimum polarization for a certain Hamiltonian, defining a ground state of the system. This system may be disrupted and contain additional information by the inversion of two narrow bits 0 and 1 .

We show that this inversion is made only by means of Alexander's T1 transformations (see Figure 2), which provides a number of dynamic invariant systems, and indirectly, ownership of causal invariance discussed by Wolfram (Wolfram, 2002). The T1 transformation is intuitively the simplest, most realistic, most economic possible evolution of a trivalent graph. It applies to a connection between two nodes, and involves two other neighbors of each of these nodes. Of these 6 nodes forming the face of an $\mathrm{H}$ if the connection is in horizontal view, the T1 transformation rotates H 90 degrees. This can be done on a continuous basis through a transient state in which a tetravalent node 5 is at the center of an X shaped figure (Figure 2 when nodes 5 and 6 are merged).

At each connection, we can apply two different transformations T1, according to one of the first neighboring node is found connected to a second neighbor node, or the other.

If the graph is locally planar, one T1 transformation retains planarity, and the other does not keep, thereby determining an implicit one that will be applied.

\subsubsection{Disrupting}

At level 1 (see Figure 1), a disturbance is the inversion of values 0 and 1 of two bits carried by two nodes, either neighbor (at distance 0 ), or narrow (at distance 1 , separated by a node).

$$
\begin{aligned}
& 1-0 \leftrightarrow 0-1 \\
& 1-1-0 \leftrightarrow 0-1-1,1-0-0 \leftrightarrow 0-0-1 \text { (Figure 4) }
\end{aligned}
$$

2.1.3 Dimension of the System as a Space

The dimension of the graph depends on the chosen scale. We distinguish three levels:

Ultra-local dimension: around a node, a trivalent graph is of dimension 2 (Ambjørn, Jurkiewicz \& Loll, 2004) (Carlip) considered the local restriction of a hexagonal lattice.

Local scale: within a super node, the structure is that of a binary tree graph whose dimension is infinite, because the number of neighbors at a distance $\mathrm{r}$ increases as $2^{r}$, and therefore more quickly than $r^{d}$ for any finite dimension $d$.

Global dimension: imposed by the regular system chosen (2 with a grid, 4 with hyperdiamond (Aschheim, 2009 b), $n-1$ with a simplex...)

\subsection{Global Topology of Space}

The global topology is flat if the network is chosen infinite.

It becomes curved if the network is closed; as for example in n-dimensional Torus.

Closure can imply a shift if a line does not meet itself after a tour, but a neighbor, like the non-commutative torus.

Closure may reverse the orientation, forming a Moebius strip, a Klein bottle, or other not oriented spaces (Luminet, 2001).

\subsection{Lattice Regularity}

\subsubsection{Regular Lattice}

We can apply our model to any regular lattice, like the square lattice, the checkerboard lattice, or even lattices in the A, D, E, F families.

We can further investigate applying it to quasi-regular lattices: Quasi-crystal (Sadoc \& Mosseri, 1997), Penrose tiling (cf. (Connes, 1990)).

\subsubsection{Almost-regularity}

We can expand the set of acceptable models to almost-regular lattice, defined as regular lattices admitting a number of irregularities $<n^{(d-l)}$ for a graph of $n^{d}$ nodes and global dimension $d$.

\subsubsection{Local Regularity}

As a special case of almost-regularity, we can build models which physically correspond to the structure of natural crystals. They are admitting irregularities on the surfaces of dimension $d-1$; locally regular but globally 
isotropic. The space is broken into pieces of irregular crystals which themselves are regular but not parallel to each other.

It will then be useful to study the dynamic behavior to inter-crystalline walls. From a mathematical point of view, the almost-regularity and local regularity are similar because they induce the same small number of irregularities with respect to the global regularity necessary.

\subsection{Intrinsic Space}

The fundamental advantage of the graphiton model is its creation of intrinsic space. The system from the outside is a graph whose nodes do not coordinate in any space (unlike all previous approaches to discretization of physics). But thanks to the encoding of information in the topology of this graph, the model finds the characteristics necessary for a metric space, beyond the mere internal metrics of the graph, which alone would be insufficient. The graphiton model is an information system capable of emulating a space. And a rather rich area because it is Riemannian (Penrose, 2005) and admits a local curvature, it can be Ricci-flat, where, in the absence of disturbance, any node at level 2 is topologically equivalent (Aschheim, 2010). And most importantly, local disturbances linearly induce a curvature, then a tilt of geodesics at any point in space, forming a gravitational field. This field is attracted towards disturbances that are at its origin, and is additive; these are necessary conditions for a gravitational field reflecting the effect of mass disruption. So this space is quite a natural framework for a theory of general relativity. But if space is locally anisotropic as directions are marked by the regular network, the gravitational induced field remains isotropic and not focused only along the directions of the network. Thus a movement in the network in response to the gravitational field can follow any direction not subject to the directions of the network.

\subsection{Space as a Network}

The general idea that the ultimate nature of space is a trivalent graph and some topological characteristics of this graph encode a mass effect, with a result on the curvature and the possibility of calculating a Ricci curvature and the Einstein field equation (Penrose, 2005) was developed by Wolfram in (Wolfram, 2002).

The fundamental contribution we make to this concept and which allows an effective implementation is to effectively place a layer of information in the graph at level 1, associated with the regular structure at level 2. This layer allows information to move intrinsically within the graph at a selected distance in both directions (2 directions for each dimension), and calculate the gravitational field and the Ricci tensor (Penrose, 2005). In the most random graph models proposed by Wolfram, these calculations were impossible and the notions of Ricci tensor couldn't be defined clearly. In other discrete models based on regular arrays, the authors postulated the existence of all space, locally Euclidean and continuous extrinsic to their model, in which they plunged their model to a metric and coordinates. What is less well founded and elegant than the concept of Wolfram! We have therefore in the extreme elegance of the model suggested by Wolfram, the only model of intrinsic system which does not need to be plunged into another space to be calculated. But we overcome its lack of metrics by imposing constraints of regularity and topological encoding of the local orientation.

\section{Mathematical Formalism}

We give a formal description of our graphiton model.

\subsection{Definitions}

Definition 1: rank r binary tree

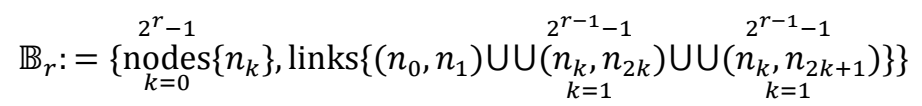

Examples are given in Figure 5 for $\mathbb{B}_{3}$ graph and its subtree from $\boldsymbol{n}_{\mathbf{2}}$.

Definition 2: descendants is a set of nodes descendants from one node

$$
\text { descendants }\left(n_{k} \text { in } \mathbb{B}_{r}\right):=\left\{\begin{array}{c}
\text { if } k<2^{r-1}: n_{k} U \operatorname{descendants}\left(n_{2 k} \operatorname{in} \mathbb{B}_{r}\right) U \operatorname{descendants}\left(n_{2 k+1} \operatorname{in} \mathbb{B}_{r}\right) \\
\text { else: } n_{k}
\end{array}\right.
$$

Definition 3: subtree is the branch emerging from a node

$$
\operatorname{subtree}\left(n_{k} \text { in } \mathbb{B}_{r}\right):=\mathbb{B}_{r} \cap \operatorname{descendants}\left(n_{k} \text { in } \mathbb{B}_{r}\right)
$$

Definition 4: an operator $\Xi$ glues three binary trees (of the 4 subtrees) to build a triple-binary trivalent graph

$$
\mathbb{T}_{r}=\Xi\left(\mathbb{B}_{r}\right) \operatorname{by}\left\{\begin{array}{l}
\text { removenodes }\left\{n_{3}, \text { descendants }\left(n_{6}\right)\right\} \text { and their links } \\
\text { addlinks }\left(n_{2}, n_{0}\right)\left(n_{0}, n_{5}\right)\left(n_{1}, n_{7}\right), \text { removelink }\left(n_{2}, n_{5}\right)
\end{array}\right.
$$


A triple-binary trivalent graph is depicted in Figure 6 with 24-nodes module $\mathbb{T}_{\mathbf{5}}=\boldsymbol{\Xi}\left(\mathbb{B}_{\mathbf{5}}\right)$, rank 5 , with 12 leaves.

Definition 5: we define the binary decomposition of an integer

$$
\beta(K):=\left\{b_{i, K}\right\} \in\{0,1\}^{\left\lfloor 1+\frac{\log K}{\log 2}\right\rfloor} / K=\sum_{i=0}^{\left\lfloor\frac{\log K}{\log 2}\right\rfloor} b_{i, K} 2^{i}
$$

Definition 6: we define the frequency of a quaternion

$$
\omega(x):=\frac{1}{\pi} \operatorname{Arc} \operatorname{Cos}(\operatorname{Re}(x))
$$

Definition 7: we define the logarithm of a quaternion from its frequency

$$
\log (x)=\log (/ x /)+\pi \omega(x) \frac{\operatorname{Im}(x)}{/ \operatorname{Im}(x) /}
$$

Definition 8: we define a family of $\mathrm{r}$ couples of a frequency and an imaginary direction

$$
\Omega_{r}:=\left\{\left\{\omega_{k}, u_{k}\right\}\left|k \in \mathbb{N} \cap\left[0, r\left[, \omega_{k} \in\right] 0,1\right], u_{k}=\operatorname{Im}\left(u_{k}\right),\right| u_{k} \mid=1\right\}
$$

Definition 9: we define a series of increasing products

$$
\forall K \in \mathbb{N} \cap\left[0,2^{r}\left[, \zeta\left(K, \Omega_{r}\right):=\eta\left(\beta(K), \Omega_{r}\right)=\prod_{i=0}^{r-1} \exp \left(b_{r-1-i, K} 2 \pi \omega_{i} u_{i}\right)\right.\right.
$$

Definition 10: we define a sample family, for $\mathrm{r}=6$

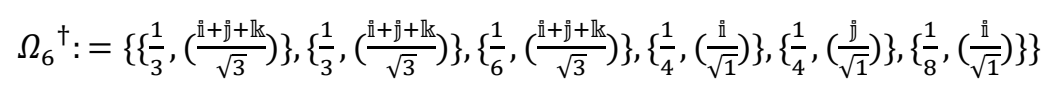

Figure 7 shows the simplest closed lattice of triple binary trees with $\boldsymbol{\Omega}_{6}{ }^{\dagger}$ is made of 2 modules and has 144 connections and 96 nodes.

Definition 11: we define a unitary diagonal quaternion

$$
\text { 떼: }=\left(\frac{i+1+\tilde{p}+\mathbb{k}}{\sqrt{3}}\right)
$$

Definition 12: we define a suite of quaternions

$$
\begin{aligned}
& \zeta\left(K, \Omega_{6}^{\dagger}\right):=\left(\left(\left(\left(\exp \left(b_{5, K} \frac{2 \pi}{3} \pi\right) \exp \left(b_{4, K} \frac{2 \pi}{3} \pi\right)\right) \exp \left(b_{3, K} \frac{2 \pi}{6} \text { 匹ा }\right)\right)\right.\right. \\
& \left.\left.\exp \left(b_{2, K} \frac{2 \pi}{4} \mathfrak{I}\right)\right) \exp \left(b_{1, K} \frac{2 \pi}{4} \mathfrak{\rrbracket}\right)\right) \exp \left(b_{0, K} \frac{2 \pi}{8} \mathfrak{I}\right)
\end{aligned}
$$

Definition 13: a field $\mathbb{E}$ is defined at each node, having a quaternionic value computed using two functions $\omega$ and $\zeta$ and one set of constants $\Omega_{r}$

$$
\begin{gathered}
\forall K \in \mathbb{N} \cap\left[0,2^{r+1}\left[, \forall n_{k} \in \operatorname{nodes}\left(\mathbb{B}_{r+1}\right),\right.\right. \\
\quad \text { if }<<2^{r}: \mathbb{E}\left(n_{2 k}\right) \\
\mathbb{E}\left(n_{k}\right):=\left\{\begin{array}{c}
\text { else: } \omega\left(\zeta\left(K-2^{r}, \Omega_{r}\right)\right) \frac{\operatorname{Im}\left(\zeta\left(K-2^{r}, \Omega_{r}\right)\right)}{\left|\operatorname{Im}\left(\zeta\left(K-2^{r}, \Omega_{r}\right)\right)\right|}
\end{array}\right.
\end{gathered}
$$

Definition 14: we define a reordering function (see Figure 8)

$$
\alpha(K):=\left(\left(K-2^{\left\lfloor\frac{\log K}{\log 2}\right\rfloor-1}+5 * 2^{\left\lfloor\frac{\log K}{\log 2}\right\rfloor-3}\right)\left[6 * 2^{\left\lfloor\frac{\log K}{\log 2}\right\rfloor-3}\right]-2^{\left\lfloor\frac{\log K}{\log 2}\right\rfloor-2}\right)\left[2^{\left\lfloor\frac{\log K}{\log 2}\right\rfloor}\right]+2^{\left\lfloor\frac{\log K}{\log 2}\right\rfloor-1}
$$

Definition 15: we define the lattice of connected triple-binary graphs, modulo $x_{0}$

$$
\mathbb{L}\left(\mathbb{T}_{r+1}, x_{0}\right):=\bigcup\left(\mathbb{T}_{r}(x)\right) \cup U\left(n_{k}\left(\mathbb{T}_{r+1}(x)\right), n_{\alpha(k)}\left(\mathbb{T}_{r+1}\left(x+2_{k}^{\frac{k[2]}{2}} \exp \left(\mathbb{E}\left(n_{k}\left(\mathbb{T}_{r+1}(x)\right)\right)\right)\left[x_{0}\right]\right)\right)\right)
$$

Definition 16: we define a lattice condition

Lattice condition on $\mathbb{E}$ (therefore on $\left.\Omega_{r}\right): \forall n_{k} \in \operatorname{nodes}\left(\mathbb{T}_{r+1}\right), k 2^{r}, k<$

$$
11 * 2^{r-3}, \mathbb{R}^{4}\left(2^{\frac{2+k[2]}{2}} \exp \left(\mathbb{E}\left(n_{k}\left(\mathbb{T}_{r+1}(x)\right)\right)\right)\right) \in \mathbb{N}^{4}
$$


Theorem 1: Lattice condition (16) holds for $\Omega_{6}^{\dagger}$

Demonstration: The values taken by $\mathbb{E}$ on the 48 leaves are the 24 units of the Hurwitz integer, forming the binary tetrahedral group, and their products by (1+i). They are also coordinates of the 24-cell (Boole Stott, 1910) and its dual scaled by square root of two (see Table 1). They form the integer lattice F4, of the roots of Lie Algebra 4.

A large family of graphiton models can be built by varying $\mathrm{r}, \Omega_{r}$ and $x_{0}$, while (16) holds.

Figure 9 shows the second simplest closed lattice of triple binary trees with $\boldsymbol{\Omega}_{\mathbf{6}}{ }^{\dagger}$ is made of 32 modules and has 2304 connections.

\section{Perturbation Tolerance}

Definition 17: we define a bit value at each node

$$
\forall K \in \mathbb{N} \cap\left[0,2^{r}\left[, \forall n_{k} \in \operatorname{nodes}\left(\mathbb{T}_{r+1}\right), \mathbb{I}\left(n_{k}\right):=k\right.\right.
$$

Topological bits in a triple binary tree according to our method preserves the intrinsic geometry. The center of the super node is determined as the single loop of size 4 . Associated with the adjacent single triangle they form the central triangle of three nodes respectively informed of bits 0,0 and 1 , and each connected to a first node of binary tree associated respectively with 0,1 and 1 . Thus, each tree root is characterized by a binary code on two different bits, and then the subsequent nodes of the branches at each level are identified by 0 and 1 . And the 24 leaves of three binary trees of rank 6 are characterized by a 5-bit identifier (Femmam, 1994). The Lattice condition Equation (16) expresses that the identifiers of two connected leaves issued from two connected triple trees have binary identifiers correlated by the function $\alpha$ which introduces redundant information and thus tolerance to disturbance.

\section{Conclusion}

In the field of artificial intelligence, attempts at architectures of neural networks were conducted confidentially with Hyperdiamond and Hypercube structures. Indeed, given the nature of space-time is linked to the four-dimensional geometry, spatial artificial intelligence, even in space-time, has good reason to rely on these geometric structures. We believe that graphiton models deserve to be studied in this area.

Pancake and hypercube networks, hypercube and in particular the cross hypercube (Tsai, Fu, \& Chen, 2008) are the subject of active research to optimize the reliability, the transmission delay, rate and energy consumption in communication networks, particularly the new networks with dynamic topology.

Our graphiton models can be considered as hybrid networks. They seem particularly interesting when the large-scale infrastructure is fixed, but only on a small scale nodes in the network connect or disconnect dynamically. This behavior corresponds to swap bit by the T1 transformations (as shown in Figure 4), local changes in the network structure. We believe that the imminent arrival of the Internet of Things and the increasing use of RFID tags, and integrated NFC antennae in mobile phones will result in the emergence of hybrid physical network. Graphiton models could be useful in the simulation and management of these new hybrid networks. Our main result is that these models define an information structure encoding both the program and data, including the hardware would be a local graph operator analyzing the surrounding environment and deciding whether or not to make a T1 topological transformation. This dynamic graph is a promising new application in artificial intelligence and the optimization of networks.

It was clarified that the proposed architecture is suitable for the production of four-dimensional neural networks, suitable for recognition of $3 \mathrm{D}$ trajectories?

The application for RFID tags is dynamically linked to a Smartphone equipped with detectors and a geo-location system, this provides to process the dynamics space-time of this information. RFID tags would be modeled by empty supernodes of our hyperdiamond network activated when detected. The features of this application will be discussed in the coming work.

\section{References}

Alexander, J. W., \& Briggs, G. B. (1926). On types of knotted curves. Annals of Math, 28(1-4), 562-586. http://dx.doi.org/10.2307/1968399

Ambjørn, J., Jurkiewicz, J., \& Loll, R. (2004). Emergence of a 4D World from Causal Quantum Gravity. Phys. Rev. Letters. http://dx.doi.org/10.1103/PhysRevLett.93.131301 
Aschheim, R. (2008). Bitmaps for a Digital Theory of Everything. Presented at the 2008 Midwest NKS conference, Indiana University, Bloomington. [Online] Available: http://www.cs.indiana.edu/ $\sim$ dgerman/2008midwestNKSconference/rasch.pdf

Aschheim, R. (2009a). From NKS to E8 symmetry, a description of the universe. JOUAL'09, CNR, Pisa, Italy.

Aschheim, R. (2009b). Hyperdiamant. From the earth to the stars symposium, Metz, France. Available on DVD, edited by Ars Mathematica.

Aschheim, R. (2010). Graphitation, digital relativity. Poster presented at the 2010 NKS Summer School, Vermont University, Burlington, VT.

Boole Stott, A. (1910). Geometrical deduction of semiregular from regular polytopes and space fillings. Verhandelingen der Koninklijke Akademie van Wetenschappente Amsterdam, 11(1), 3-24.

Carlip, S. The Small Scale Structure of Space time. In Foundations of Space and Time, edited by George Ellis, Jeff Murugan, Amanda Weltman: Cambridge University Press. [Online] Available: http://arxiv.org/abs/1009.1136

Chamseddine, A., \& Connes, A. (2010). Noncommutative geometry as a framework for unification of all fundamental interactions including gravity. Part I. Fortschritte der Physik, 58, 553-600. http://dx.doi.org/10.1002/prop.201000069

Connes, A. (1990). Géométrie non commutative. Inter Editions, Paris.

De Ockam, G. (a). Quaestiones et decisiones in quatuor libros Sententiarum cum centilogio theologico li vre II (1319). [Online] Available: http://fondotesis.us.es/fondos/libros/256/553/quaestiones-et-decisiones-in-i v-libros-sententiarum-cum-centilogio-theologico/?desplegar $=2708$

De Ockam, G. (b). Dialogus. Part 3, tract 2, book 3, chapter17, (1340). [Online] Available: http://www.britac.ac.uk/pubs/dialogus/t32d3c3.html

Femmam, S., Zerarka, M.F., Benakila, I., \& Ouahabi, A. (2012). New Approach Construction for Wireless ZigBee Sensor Based on Embedding Pancake Graphs. To appear in Network and Communication Technologies, 1(1).

Femmam, S. (1994). Study and design of Lattice networks algorithms applied to adaptive prediction filters. Master thesis, University of Annaba.

Luminet, J.P. (2001). L'univers chiffonné. Paris, Fayard.

Konopka, T., Markopoulou, F., \& Severini, S. (2008). Quantum Graphity: A Model of emergent locality. Physics Review. http://dx.doi.org/10.1103/PhysRevD.77.104029

Markopoulou, F, \& Smolin, L. (2004). Quantum Theory from Quantum Gravity. Quantum Physics. http://dx.doi.org/10.1103/PhysRevD.70.124029

Penrose, R. (2005). The Road to Reality: A Complete Guide to the Laws of the Universe. Knopf.

Sadoc, J.F., \& Mosseri, R. (1997). Frustration géométrique. Paris, Eyrolles.

Tsai, P.-Y., Fu, J.-S. \& Chen, G.-H. (2008). Edge-fault-tolerant Hamilton city of pancake graphs under the conditional fault model. Theoretical Computer Science, 409, 450-460. http://dx.doi.org/10.1016/j.tcs.2008.09.015

Wolfram, S. (2002). A New Kind of Science. Champaign, IL: Wolfram Media. 
Table 1. Values of $\mathbb{E}\left(n_{k}\right)$ field

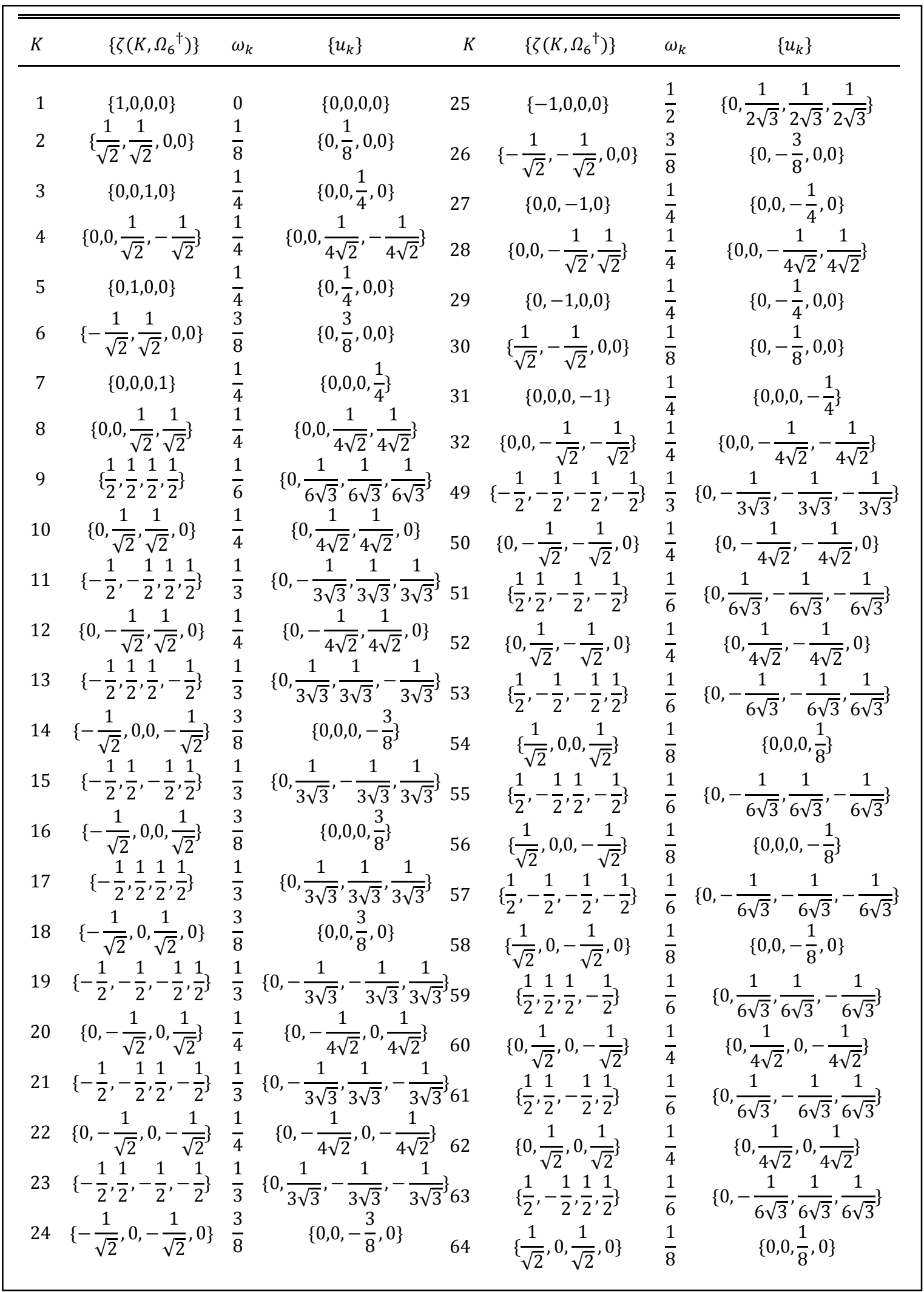

Field values for the 48 leaves of the triple binary tree $\mathbb{T}_{7}=\Xi\left(\mathbb{B}_{7}\right)$ based on $\Omega_{6}{ }^{\dagger}$, pointing toward linked supernode, such that opposite leaves have opposite field. 


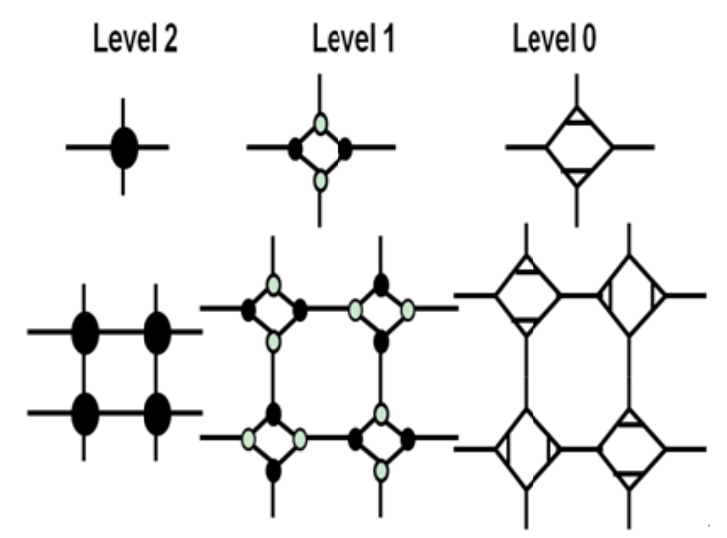

Figure 1. Three-level view of a) a simple node, b) a sub-graph. At level 2 the graph is not trivalent At level 1, nodes are added so the graph becomes trivalent, and nodes hold a bit.

At level 0, 1-valued nodes are replaced by triangles.
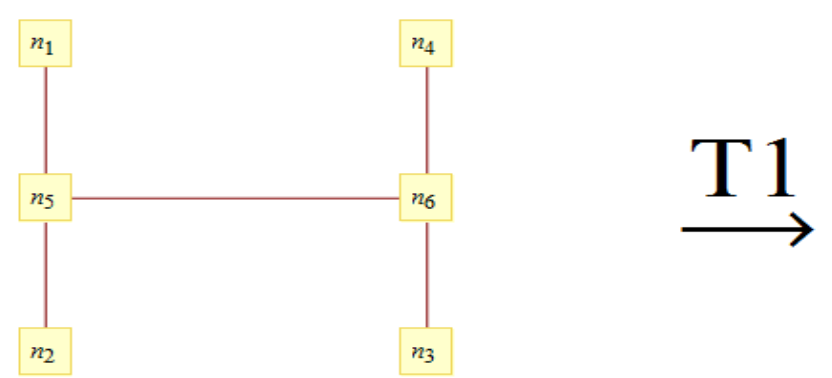

Figure 2. Elementary move: T1 inversion
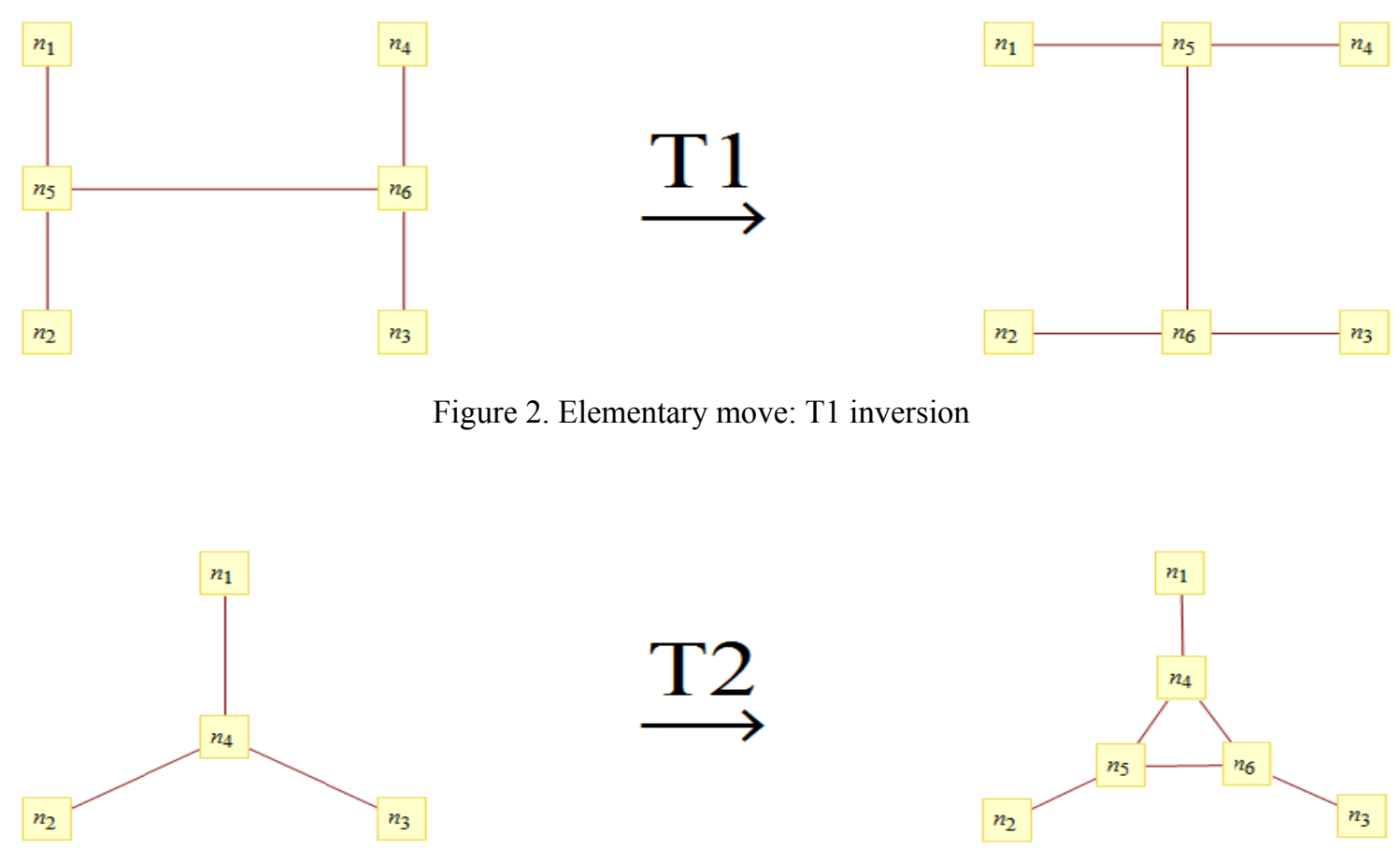

Figure 3. Elementary move: T2 insertion

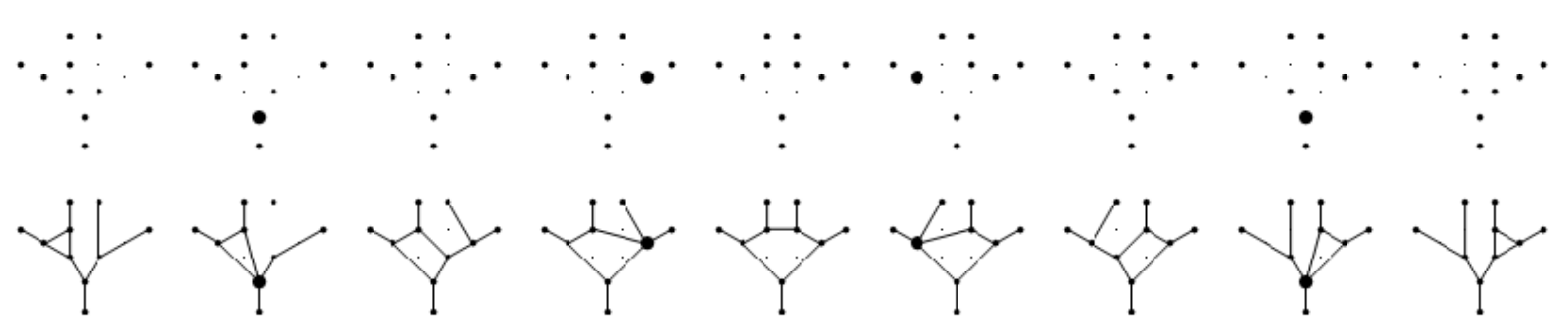

Figure 4. Bit inversion $1-0-0 \leftrightarrow 0-0-1$ as a sequence of four Alexander T1 moves

(The transient four-valent $\mathrm{X}$-state is enhanced by a bigger dot). 

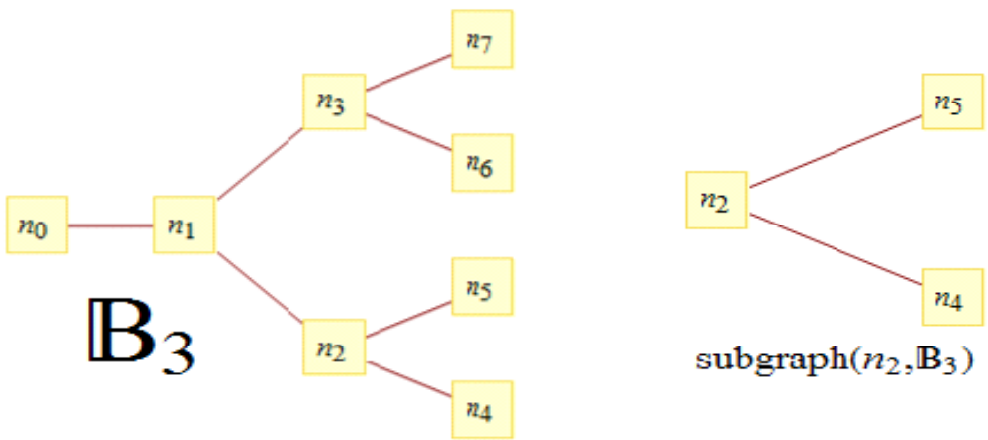

$\operatorname{subgraph}\left(n_{2}, \mathbb{B}_{3}\right)$

Figure 5. Examples: a $\mathbb{B}_{3}$ graph (left), its subtree from $\boldsymbol{n}_{\mathbf{2}}$ (right)

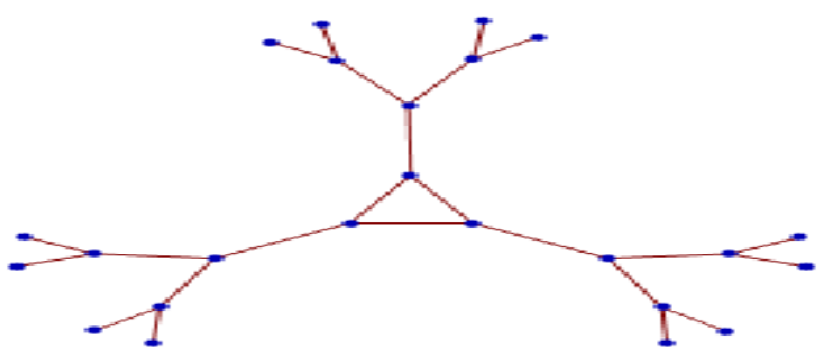

Figure 6. 24-nodes module $\mathbb{T}_{5}=\boldsymbol{\Xi}\left(\mathbb{B}_{5}\right)$, rank 5 , with 12 leaves

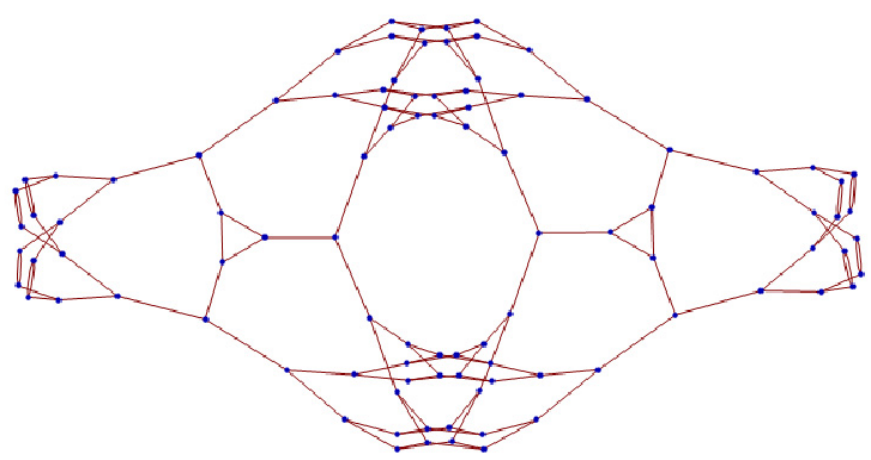

Figure 7. The simplest closed lattice of triple binary trees with $\boldsymbol{\Omega}_{\mathbf{6}}{ }^{\dagger}$ is made of 2 modules and has 144 connections and 96 nodes 


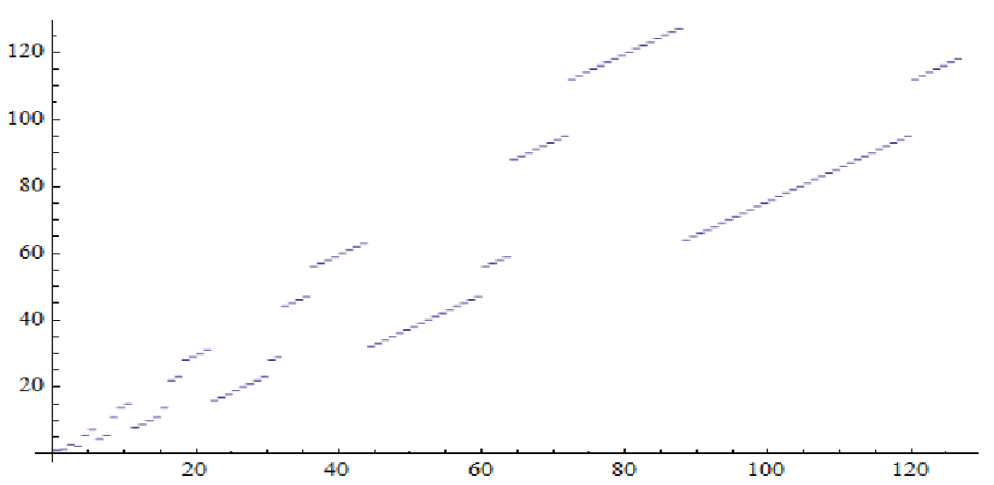

Figure 8. Plot of the $\boldsymbol{\alpha}$ (n) function (the values of $\boldsymbol{\alpha}$ versus n)

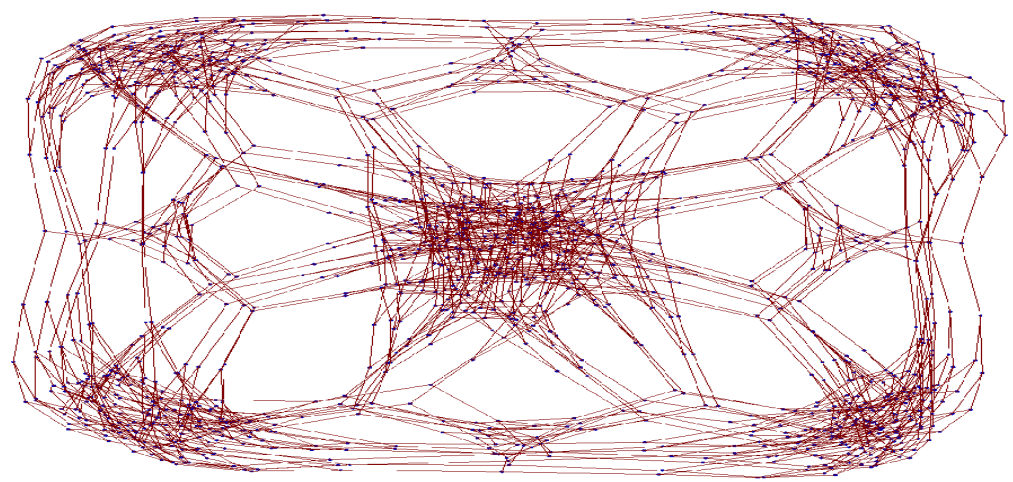

Figure 9. The second simplest closed lattice of triple binary trees with $\boldsymbol{\Omega}_{\mathbf{6}}{ }^{\dagger}$ is made of 32 modules and has 2304 connections 